\title{
Beyond NURBS - Efficient CAD-integrated Isogeometric Analysis
}

\author{
T. Oberbichler ${ }^{\dagger *}$, R. Wüchner ${ }^{\dagger}$ and K.-U. Bletzinger ${ }^{\dagger}$ \\ ${ }^{\dagger}$ Chair of Structural Analysis, Department of Civil, Geo and Environmental Engineering \\ Technical University of Munich (TUM) \\ Arcisstraße 21, 80333 Munich, Germany \\ * e-mail: thomas.oberbichler@tum.de
}

\begin{abstract}
The integration of analysis tools in computer aided design (CAD) enables structures to be generated and explored intuitively. To achieve a high degree of interactivity, the use of natural CAD geometric parametrization - for example NURBS - is also desirable at the analysis stage [1]. It is important for this to be done in a computationally efficient manner, to speed up parametric design iterations. However, the analysis of free-form geometries based on NURBS is computationally costly due to the high polynomial degree. Beyond NURBS, modern CAD systems provide other descriptions of freeform geometries, such as discrete meshes or subdivision surfaces. To perform various types of analysis with different geometric descriptions, it is necessary to generalize the process of CAD-integrated isogeometric analysis (IGA) while also increasing the computational speed.
\end{abstract}

To address this issue, we present a new, efficient, and modular approach for implementing CADintegrated analysis. It merges the concepts of the adjoint method with algorithmic differentiation to achieve a clean separation between mechanics and geometry [2]. Extracting the NURBS from the mechanics enables more efficient implementation with tighter integration of the analysis tool in a CAD system, which allows extensive interaction with the physical model. The same mechanical formulation can be combined with different geometric descriptions, and vice versa. A feature-rich digital toolbox can be derived from a set of highly optimized mechanical and geometric building blocks.

We present this concept for a range of mechanical element types and geometric parameterizations. The method can be employed for classic structural analysis as well as in form-finding and the constraintdriven design of free-form geometries.

\section{REFERENCES}

[1] T.J.R. Hughes, J.A. Cottrell and Y. Bazilevs, Isogeometric analysis: CAD, finite elements, NURBS, exact geometry and mesh refinement, Computer Methods in Applied Mechanics and Engineering, 194, 4135-4195 (2005).

[2] T. Oberbichler, R. Wüchner and K.-U. Bletzinger, Efficient Computation of Nonlinear Isogeometric Elements using the Adjoint Method and Algorithmic Differentiation, Computer Methods in Applied Mechanics and Engineering, 381, 113817 (2021). 